\title{
Estimation of Total Energy Load of Building Using Artificial Neural Network
}

\author{
Rajesh Kumar $^{1}$, RK Aggarwal ${ }^{2, *}$ and J D Sharma ${ }^{1}$ \\ ${ }^{1}$ Department of Physics, Shoolini University, Bajhol, Solan 173212 (India) \\ ${ }^{2}$ Department of Environmental Science, Dr Y S Parmar University of Horticulture \& Forestry, Nauni (Solan), 173230 (India) \\ *Corresponding Author: rajeev1792@rediffmail.com
}

Copyright $(2013$ Horizon Research Publishing All rights reserved.

\begin{abstract}
This paper explores total heat load and total carbon emissions of a six storey building by using artificial neural network (ANN). The energy performance in buildings is influenced by many factors such as ambient weather conditions, building structure and characteristics, the operation of sub-level components like lighting and HVAC systems, occupancy and their behavior. This complex situation makes it very difficult to accurately implement the prediction of building energy consumption. The calculated total load was 2.09 million $\mathrm{kW}$ per year. ANN application showed that data was best fitted for the regression coefficient of 0.99812 with best validation performance of 1312.5203 in case of total heat load. To meet out this energy demand fuel options are presented along with their cost and carbon emission and necessary measures has been given.
\end{abstract}

Keywords Artificial neural network, energy requirement, solar heat gain, internal gain, ventilation losses, carbon emission

\section{Introduction}

Himachal Pradesh is located in north India with Latitude $30^{\circ} 22^{\prime} 40^{\prime \prime} \mathrm{N}$ to $33^{\circ} 12^{\prime} 40^{\prime \prime} \mathrm{N}$, Longitude $75^{\circ} 45^{\prime} 55^{\prime \prime} \mathrm{E}$ to $79^{\circ}$ $04^{\prime} 20^{\prime \prime} \mathrm{E}$, height (from mean sea level) 350 meter to 6975 meter and average rainfall $1300 \mathrm{~mm}$. For our study we have taken a building in Solan district which is located between the longitudes 76.42 and 77.20 degree and latitudes 30.05 and 31.15 degree north the elevation of the district ranges from 300 to 3,000 meter above sea level. The winters during six months (October to March) are severe and people use electricity (provided on subsidized rates) and conventional fuels (wood, LPG and coal). The summers during six months (April to September) people use electricity (provided on subsidized rates) to lower down the temperature. These results are burden on already depleting conventional fuels and same time causing emission of $\mathrm{CO}_{2}$ and global warming. The other option to meet out energy requirement is solar passive technologies. This requires measured data of solar radiation which is not available in the state. This can be estimated by using various models on the basis of sunshine hour or temperature. The mean hourly values of such data for various places in India are available in the handbook [1]. As in statistical methods we have to deal with higher level of mathematics. Due to tough calculations, the probability of error is more. Evaluation, estimation and prediction are often done using statistical packages such as SAS, SPSS, GENST AT etc. Most of these packages are based on conventional algorithms such as the least square method, moving average, time series, curve fitting etc. The performances of these algorithms are not robust enough when the data set becomes very large. This approach is very much time as well as mind consuming. Therefore ANN is much better than these methods. Neural networks have the potential for making better, quicker and more practical predictions than any of the traditional methods. They can be used to predict energy consumption more reliably than traditional simulation models and regression techniques. Artificial Neural Networks are nowadays accepted as an alternative technology offering a way to tackle complex and ill-defined problems. They are not programmed in the traditional way but they are trained using past history data representing the behaviour of a system.

ANNs are the most widely used artificial intelligence models in the application of building energy prediction. In the past twenty years, researchers have applied ANNs to analyze various types of building energy consumption in a variety of conditions, such as heating/cooling load, electricity consumption, sub-level components operation and optimization, estimation of usage parameters. In 2006, Kalogirou did a brief review of the ANNs in energy applications in buildings, including solar water heating systems, solar radiation, wind speed, air flow distribution inside a room, pre-diction of energy consumption, indoor air temperature, and HVAC system analysis [2]. After that he used back propagation neural networks to predict the required heating load of buildings [3]. The model was trained on the consumption data of 225 buildings which vary largely 
from small spaces to big rooms. Ekici et al. used the same model to predict building heating loads in three buildings [4]. The training and testing datasets were calculated by using the finite difference approach of transient state one-dimensional heat conduction. Olofsson et al. developed a neural network which makes long-term energy demand (the annual heating demand) predictions based on short-term (typically 2-5 weeks) measured data with a high prediction rate for single family buildings [5]. Reference [6] used a back propagation neural network to predict cooling demand in a building. In their work, a global optimization method called modal trimming method was proposed for identifying model parameters. Kreider et al. reported results of a recurrent neural network on hourly energy consumption data to predict building heating and cooling energy needs in the future, knowing only the weather and time stamp [7]. Based on the same recurrent neural network, [8] predicted the cooling load of three office buildings. Reference [9] used neural networks for the prediction of the energy consumption of a passive solar building where mechanical and electrical heating devices are not used. Considering the influence of weather on the energy consumption in different regions a back propagation neural network to predict building's heating and cooling load in different climate zones represented by heating degree day and cooling degree day [10]. Reference [11] used a neural network to predict energy consumption for office buildings with day-lighting controls in subtropical climates. Neural network can be used to estimate appliance, lighting and space cooling energy consumption and it is also a good model to estimate the effects of the socio-economic factors on this consumption in the Canadian residential sector [12]. Reference [13] did Short Term Load Forecasting using ANN Technique. Kreider et al. reported results of recurrent neural networks on hourly energy consumption data [7]. Reference [4] predicted building heating loads without considering climatic variables. Reference [14] studied how statistical procedures can improve neural network models in the prediction of hourly energy loads.

\section{Method and Material}

The six storey administrative block of Shoolini University building at Bajhol-Solan (HP) had been taken for the study, which worked for seven hours during a day time. The dimensions were length $45 \mathrm{~m}, 15 \mathrm{~m}$ wide and $18 \mathrm{~m}$ in height. The winters during six months (October to March) are severe at Solan and people use electricity (provided on subsidized rates) and conventional fuels (wood and coal) for heating and cooking purposes. The neural fitting tool (nftool) of neural network of MATLAB Version 7.11.0.584 (R2010b) with 32-bit (win 32) had been used. The temperature and humidity of the building had been measured by using 'Thermo Hygrometer' for four consecutive years between 2009 -2012. The floorwise building data had been measured with measuring tape as has been depicted in Table 1, Table 2, Table 3, Table 4, Table 5 and Table 6 . The other required data had been taken from NASA website. 
Table 1. Dimensions of ground floor

\begin{tabular}{|c|c|c|c|c|c|c|c|c|c|c|}
\hline \multirow[b]{2}{*}{ S N } & \multicolumn{4}{|c|}{ Room } & \multicolumn{3}{|c|}{ Window } & \multicolumn{3}{|c|}{ Door } \\
\hline & No & Type & $\begin{array}{c}\text { Dimensions } \\
\text { lbh* } \\
\text { (In Foot) } \\
\end{array}$ & Orientation & Quantity & $\begin{array}{c}\text { Dimensions } \\
\text { lbh* } \\
\text { (In Foot) } \\
\end{array}$ & Orientation & Quantity & $\begin{array}{l}\text { Dimensions } \mathrm{lb}^{* *} \\
\quad \text { (In Foot) }\end{array}$ & Orientation \\
\hline \multirow{2}{*}{1} & \multirow{2}{*}{1} & \multirow{2}{*}{ VC Office } & \multirow{2}{*}{$18 \times 8 \times 10$} & \multirow{2}{*}{ - } & 1 & $4 \times 9$ & S & \multirow{2}{*}{1} & \multirow{2}{*}{$3 \times 9$} & \multirow{2}{*}{$\mathrm{N}$} \\
\hline & & & & & 1 & $18 \times 6$ & $\mathrm{~N}$ & & & \\
\hline \multirow{2}{*}{2} & \multirow{2}{*}{2} & \multirow{2}{*}{ VC Room } & \multirow{2}{*}{$25 \times 18 \times 10$} & \multirow{2}{*}{ - } & 2 & $4 \times 9$ & S & 1 & $3 \times 9$ & $\mathrm{~N}$ \\
\hline & & & & & 1 & $10 \times 4$ & $\mathrm{~N}$ & 1 & $3 \times 9$ & $\mathrm{~W}$ \\
\hline 3 & - & Balcony & $36 \times 20 \times 10$ & - & - & - & - & - & - & - \\
\hline 4 & - & Pantry & $20 \times 8 \times 10$ & - & 1 & $15 \times 7$ & S & 1 & $3 \times 9$ & S \\
\hline 5 & - & Toilet VC & $8 \times 6 \times 10$ & - & - & - & - & 1 & $2 \times 6$ & - \\
\hline \multirow{2}{*}{6} & \multirow{2}{*}{-} & \multirow{2}{*}{ Registrar Office } & \multirow{2}{*}{$30 \times 22 \times 10$} & \multirow{2}{*}{$\mathrm{N}$} & 1 & $6 \times 5$ & - & \multirow{2}{*}{1} & \multirow{2}{*}{$3 \times 9$} & \multirow{2}{*}{ S } \\
\hline & & & & & 1 & $5 \times 16$ & S & & & \\
\hline 7 & 4 & $\begin{array}{c}\text { Office } \\
\text { Associate Dean }\end{array}$ & $14 \times 10 \times 10$ & S & 1 & $4 \times 9$ & S & 1 & $3 \times 9$ & $\mathrm{~N}$ \\
\hline 8 & 5 & $\begin{array}{l}\text { Office President } \\
\text { Foundation }\end{array}$ & $14 \times 10 \times 10$ & S & 1 & $4 \times 9$ & S & 1 & $3 \times 9$ & $\mathrm{~N}$ \\
\hline 9 & 6 & Seminar Hall & $22 \times 18 \times 10$ & $\mathrm{~N}$ & 1 & $4 \times 9$ & - & 1 & $3 \times 9$ & $\mathrm{~S}$ \\
\hline 10 & 7 & Accounts Office & $25 \times 12 \times 10$ & - & 1 & $4 \times 9$ & $\mathrm{E}$ & 1 & $3 \times 9$ & S \\
\hline 11 & 8 & COF Office & $20 \times 12 \times 10$ & - & 1 & $4 \times 9$ & $\mathrm{E}$ & 1 & $3 \times 9$ & S \\
\hline \multirow{2}{*}{12} & \multirow{2}{*}{-} & \multirow{2}{*}{ Registrar Room } & \multirow{2}{*}{$25 \times 12 \times 10$} & \multirow{2}{*}{ - } & \multirow{2}{*}{1} & \multirow{2}{*}{$4 \times 9$} & \multirow{2}{*}{$\mathrm{E}$} & 1 & $3 \times 9$ & S \\
\hline & & & & & & & & 1 & $3 \times 9$ & $\mathrm{~N}$ \\
\hline 13 & - & Director Room & $20 \times 12 \times 10$ & - & 1 & $4 \times 9$ & W & 1 & $3 \times 9$ & S \\
\hline 14 & - & Balcony & $16 \times 20 \times 10$ & - & - & - & - & - & - & - \\
\hline 15 & - & Toilet Faculty & $4 \times 6 \times 10$ & - & - & - & - & - & - & - \\
\hline
\end{tabular}


Table 2. Dimensions of first floor

\begin{tabular}{|c|c|c|c|c|c|c|c|c|c|c|}
\hline \multirow[b]{2}{*}{$\mathrm{S} \mathrm{N}$} & \multicolumn{4}{|c|}{ Room } & \multicolumn{3}{|c|}{ Window } & \multicolumn{3}{|c|}{ Door } \\
\hline & No & Type & $\begin{array}{c}\text { Dimensions } \\
\text { lbh* } \\
\text { (In Foot) }\end{array}$ & Orientation & Quantity & $\begin{array}{c}\text { Dimensions } \\
\text { lbh* } \\
\text { (In Foot) }\end{array}$ & Orientation & Quantity & $\begin{array}{c}\text { Dimensions } \\
\text { lb** } \\
\text { (In Fooot) }\end{array}$ & Orientation \\
\hline 1 & - & Photo Stat Room & $12 \times 10 \times 10$ & $\mathrm{~N}$ & - & - & - & - & - & - \\
\hline 2 & - & Balcony & $20 \times 16 \times 10$ & S & - & - & - & - & - & - \\
\hline 3 & 101 & LAB (IT) & $50 \times 25 \times 10$ & $\mathrm{~N}$ & 5 & $5 \times 6$ & $\mathrm{~N}$ & 1 & $4 \times 9$ & $\mathrm{~S}$ \\
\hline 4 & 102 & Faculty Room & $28 \times 18 \times 10$ & $\mathrm{~S}$ & 2 & $6 \times 5$ & $\mathrm{~S}$ & 1 & $4 \times 9$ & $\mathrm{~S}$ \\
\hline \multirow{2}{*}{5} & \multirow{2}{*}{103} & \multirow{2}{*}{ Faculty Room } & \multirow{2}{*}{$22 \times 12 \times 10$} & \multirow{2}{*}{$\mathrm{S}$} & 1 & $6 \times 5$ & $\mathrm{~S}$ & \multirow{2}{*}{1} & \multirow{2}{*}{$3 \times 9$} & \multirow{2}{*}{$\mathrm{S}$} \\
\hline & & & & & 1 & $8 \times 6$ & $\mathrm{~N}$ & & & \\
\hline 6 & 104 & Dean Room & $22 \times 12 \times 10$ & $\mathrm{~S}$ & $\begin{array}{l}1 \\
2\end{array}$ & $\begin{array}{l}8 \times 6 \\
5 \times 6\end{array}$ & $\begin{array}{c}\text { S } \\
\text { W }\end{array}$ & 1 & $3 \times 9$ & $\mathrm{~N}$ \\
\hline 7 & - & Faculty Cabin & $30 \times 35 \times 10$ & $\mathrm{~S}$ & 4 & $4 \times 6$ & $\mathrm{~S}$ & 2 & $4 \times 9$ & - \\
\hline \multirow{2}{*}{8} & \multirow{2}{*}{-} & \multirow{2}{*}{ Seminar Hall } & \multirow{2}{*}{$50 \times 30 \times 10$} & \multirow{2}{*}{$\mathrm{N}$} & 2 & $4 \times 6$ & $\mathrm{~S}$ & \multirow{2}{*}{1} & \multirow{2}{*}{$3 \times 9$} & \multirow{2}{*}{-} \\
\hline & & & & & 4 & $4 \times 6$ & $\mathrm{~N}$ & & & \\
\hline 9 & - & Canteen & $10 \times 8 \times 10$ & $\mathrm{~S}$ & 1 & $10 \times 4$ & $\mathrm{~S}$ & 1 & $3 \times 6$ & $\mathrm{~N}$ \\
\hline 10 & - & Balcony & $25 \times 15 \times 10$ & $\mathrm{~S}$ & 1 & $4 \times 6$ & E & - & - & - \\
\hline
\end{tabular}


Table 3. Dimensions of second floor

\begin{tabular}{|c|c|c|c|c|c|c|c|c|c|c|}
\hline \multirow{2}{*}{$\mathrm{S} \mathrm{N}$} & \multicolumn{4}{|c|}{ Room } & \multicolumn{3}{|c|}{ Window } & \multicolumn{3}{|c|}{ Door } \\
\hline & No & Type & $\begin{array}{c}\text { Dimensions lbh* } \\
\text { (In Foot) }\end{array}$ & Orientation & Quantity & $\begin{array}{l}\text { Dimensions lbh* } \\
\text { (In Foot) }\end{array}$ & Orientation & Quantity & $\begin{array}{c}\text { Dimensions lb** } \\
\text { (In Foot) }\end{array}$ & Orientation \\
\hline 1 & 201 & Microbio Lab & $40 \times 20 \times 10$ & $\mathrm{~N}$ & 2 & $5 \times 6$ & $\mathrm{~N}$ & 2 & $4 \times 9$ & S \\
\hline 2 & 202 & Tissue Culture Lab & $25 \times 20 \times 10$ & S & - & - & - & 1 & $4 \times 6$ & $\mathrm{~N}$ \\
\hline \multirow{2}{*}{3} & \multirow{2}{*}{203} & \multirow{2}{*}{ Animal Lab } & \multirow{2}{*}{$40 \times 20 \times 10$} & \multirow{2}{*}{ S } & 2 & $5 \times 6$ & $\mathrm{~N}$ & 1 & $4 \times 9$ & $\mathrm{E}$ \\
\hline & & & & & 3 & $5 \times 6$ & $\mathrm{E}$ & 1 & $3 \times 6$ & $\mathrm{~N}$ \\
\hline 4 & 204 & Store & $15 \times 8 \times 10$ & $\mathrm{~S}$ & - & - & - & 1 & $4 \times 9$ & E \\
\hline \multirow{2}{*}{5} & \multirow{2}{*}{205} & \multirow{2}{*}{$\mathrm{Lab}$} & \multirow{2}{*}{$50 \times 20 \times 10$} & \multirow{2}{*}{$\mathrm{S}$} & 2 & $5 \times 6$ & S & \multirow{2}{*}{1} & \multirow{2}{*}{$4 \times 9$} & \multirow{2}{*}{$\mathrm{N}$} \\
\hline & & & & & 2 & $4 \times 6$ & $\mathrm{~S}$ & & & \\
\hline \multirow{2}{*}{6} & \multirow{2}{*}{206} & \multirow{2}{*}{$\mathrm{Lab}$} & \multirow{2}{*}{$20 \times 20 \times 10$} & \multirow{2}{*}{ S } & 2 & $5 \times 6$ & $\mathrm{~S}$ & 1 & $4 \times 9$ & $\mathrm{~N}$ \\
\hline & & & & & 1 & $5 \times 6$ & $\mathrm{~N}$ & 1 & $4 \times 9$ & $\mathrm{~S}$ \\
\hline 7 & - & Toilet She & $6 \times 8 \times 10$ & $\mathrm{~N}$ & 2 & $4 \times 2$ & $\mathrm{~N}$ & 1 & $3 \times 6$ & $\mathrm{~S}$ \\
\hline 8 & - & Store & $6 \times 8 \times 10$ & $\mathrm{~N}$ & 1 & $4 \times 5$ & $\mathrm{~N}$ & 1 & $3 \times 9$ & S \\
\hline 9 & 201 & Lecture Theatre MBA & $50 \times 20 \times 10$ & $\mathrm{~N}$ & 3 & $5 \times 6$ & $\mathrm{~N}$ & 1 & $4 \times 9$ & S \\
\hline 10 & 202 & $\begin{array}{c}\text { Lecture Theatre } \\
\text { MBA }\end{array}$ & $50 \times 20 \times 10$ & $\mathrm{~N}$ & 2 & $5 \times 6$ & $\mathrm{~N}$ & 1 & $4 \times 9$ & $\mathrm{~W}$ \\
\hline 11 & 203 & $\begin{array}{c}\text { Lecture Theatre } \\
\text { MBA }\end{array}$ & $50 \times 20 \times 10$ & $\mathrm{~S}$ & 2 & $5 \times 6$ & $\mathrm{~S}$ & 1 & $4 \times 9$ & $\mathrm{~W}$ \\
\hline \multirow{2}{*}{12} & \multirow{2}{*}{-} & \multirow{2}{*}{ Faculty Room } & \multirow{2}{*}{$18 \times 8 \times 10$} & \multirow{2}{*}{ S } & \multirow{2}{*}{2} & \multirow{2}{*}{$5 \times 6$} & \multirow{2}{*}{ S } & 1 & $3 \times 6$ & $\mathrm{~N}$ \\
\hline & & & & & & & & 1 & $3 \times 6$ & $\mathrm{~S}$ \\
\hline \multirow{2}{*}{13} & & \multirow{2}{*}{ Balcony } & \multirow{2}{*}{$20 \times 15 \times 10$} & \multirow{2}{*}{ S } & 2 & $5 \times 6$ & S & & & \\
\hline & & & & & 1 & $5 \times 6$ & $\mathrm{~W}$ & & & \\
\hline
\end{tabular}


Table 4. Dimensions of third floor

\begin{tabular}{|c|c|c|c|c|c|c|c|c|c|c|}
\hline \multirow{2}{*}{$\mathrm{S} \mathrm{N}$} & \multicolumn{4}{|c|}{ Room } & \multicolumn{3}{|c|}{ Window } & \multicolumn{3}{|c|}{ Door } \\
\hline & No & Type & $\begin{array}{l}\text { Dimensions lbh* } \\
\text { (In Foot) }\end{array}$ & Orientation & Quantity & $\begin{array}{l}\text { Dimensions lbh* } \\
\quad \text { (In Foot) }\end{array}$ & Orientation & Quantity & $\begin{array}{l}\text { Dimensions } 1 b^{* *} \\
\quad(\text { In Foot })\end{array}$ & Orientation \\
\hline \multirow{2}{*}{1} & \multirow{2}{*}{301} & \multirow{2}{*}{ BT Lab } & \multirow{2}{*}{$40 \times 15 \times 10$} & \multirow{2}{*}{$\mathrm{N}$} & 2 & $5 \times 6$ & $\mathrm{~N}$ & \multirow{2}{*}{1} & \multirow{2}{*}{$4 \times 9$} & \multirow{2}{*}{$\mathrm{S}$} \\
\hline & & & & & 3 & $5 \times 6$ & $\mathrm{~W}$ & & & \\
\hline 2 & 302 & HOD BT & $15 \times 8 \times 10$ & $\mathrm{~N}$ & 2 & $4 \times 6$ & S & 1 & $3 \times 9$ & $\mathrm{~S}$ \\
\hline \multirow{2}{*}{3} & \multirow{2}{*}{303} & \multirow{2}{*}{ Chem LAB } & \multirow{2}{*}{$40 \times 20 \times 10$} & \multirow{2}{*}{$\mathrm{N}$} & 2 & $5 \times 6$ & $\mathrm{~N}$ & 1 & $4 \times 6$ & $\mathrm{E}$ \\
\hline & & & & & 3 & $5 \times 6$ & E & 1 & $3 \times 6$ & $\mathrm{~S}$ \\
\hline 4 & 304 & Store & $6 \times 8 \times 10$ & $\mathrm{~N}$ & 1 & $4 \times 5$ & $\mathrm{~N}$ & 1 & $3 \times 9$ & $\mathrm{~S}$ \\
\hline \multirow{2}{*}{5} & \multirow{2}{*}{305} & \multirow{2}{*}{ Chem LAB } & \multirow{2}{*}{$50 \times 18 \times 10$} & \multirow{2}{*}{ - } & \multirow{2}{*}{4} & \multirow{2}{*}{$5 \times 6$} & \multirow{2}{*}{ S } & 1 & $4 \times 6$ & $\mathrm{~N}$ \\
\hline & & & & & & & & 1 & $3 \times 6$ & $\mathrm{~N}$ \\
\hline \multirow{2}{*}{6} & \multirow{2}{*}{306} & \multirow{2}{*}{ Faculty Room } & \multirow{2}{*}{$20 \times 20 \times 10$} & \multirow{2}{*}{$\mathrm{S}$} & 2 & $5 \times 6$ & $\mathrm{~S}$ & 1 & $4 \times 9$ & $\mathrm{~N}$ \\
\hline & & & & & 1 & $5 \times 6$ & $\mathrm{~N}$ & 1 & $4 \times 9$ & $\mathrm{~S}$ \\
\hline 7 & 301 & $\begin{array}{c}\text { Confrence Room } \\
\text { (MBA) }\end{array}$ & $50 \times 20 \times 10$ & - & 2 & $5 \times 6$ & $\mathrm{~N}$ & 1 & $4 \times 9$ & W \\
\hline 8 & 302 & $\begin{array}{c}\text { Confrence Room } \\
\text { (MBA) }\end{array}$ & $50 \times 20 \times 10$ & - & 3 & $5 \times 6$ & $\mathrm{~N}$ & 1 & $4 \times 9$ & $\mathrm{~W}$ \\
\hline 9 & 303 & Meditaion Room & $40 \times 25 \times 10$ & & 3 & $5 \times 6$ & $\mathrm{~N}$ & 1 & $4 \times 9$ & W \\
\hline 10 & - & Toilet $\mathrm{He}$ & $6 \times 8 \times 10$ & $\mathrm{~N}$ & 2 & $4 \times 2$ & $\mathrm{~N}$ & 1 & $3 \times 6$ & $\mathrm{~S}$ \\
\hline 11 & - & Store & $6 \times 8 \times 10$ & $\mathrm{~N}$ & 1 & $4 \times 5$ & $\mathrm{~N}$ & 1 & $3 \times 9$ & $\mathrm{~S}$ \\
\hline
\end{tabular}


Table 5. Dimensions of fourth floor

\begin{tabular}{|c|c|c|c|c|c|c|c|c|c|c|}
\hline \multirow{2}{*}{$\mathrm{S} \mathrm{N}$} & \multicolumn{2}{|r|}{ Room } & \multirow{2}{*}{$\begin{array}{l}\text { Dimensions lbh* } \\
\text { (In Foot) }\end{array}$} & \multirow{2}{*}{ Orientation } & \multicolumn{3}{|c|}{ Window } & \multicolumn{3}{|c|}{ Door } \\
\hline & No & Type & & & Quantity & $\begin{array}{c}\text { Dimensions lbh* } \\
\text { (In Foot) }\end{array}$ & Orientation & Quantity & $\begin{array}{c}\text { Dimensions lb** } \\
\text { (In Foot) }\end{array}$ & Orientation \\
\hline \multirow{3}{*}{1} & \multirow{3}{*}{-} & \multirow{3}{*}{ Canteen } & \multirow{3}{*}{$60 \times 40 \times 10$} & \multirow{3}{*}{$\mathrm{S}$} & 5 & $5 \times 6$ & $\mathrm{~S}$ & 1 & $5 \times 9$ & $\mathrm{E}$ \\
\hline & & & & & \multirow{2}{*}{5} & \multirow{2}{*}{$5 \times 6$} & \multirow{2}{*}{$\mathrm{N}$} & 1 & $3 \times 9$ & $\mathrm{~S}$ \\
\hline & & & & & & & & 1 & $4 \times 6$ & W \\
\hline \multirow{4}{*}{2} & \multirow{4}{*}{ - } & \multirow{4}{*}{ Auditorium } & \multirow{4}{*}{$60 \times 40 \times 10$} & \multirow{4}{*}{$\mathrm{S}$} & 3 & $4 \times 6$ & $\mathrm{~S}$ & 1 & $4 \times 6$ & $\mathrm{~S}$ \\
\hline & & & & & \multirow{3}{*}{1} & \multirow{3}{*}{$4 \times 6$} & \multirow{3}{*}{$\mathrm{N}$} & 1 & $4 \times 6$ & $\mathrm{E}$ \\
\hline & & & & & & & & 1 & $4 \times 6$ & $\mathrm{~W}$ \\
\hline & & & & & & & & 1 & $5 \times 6$ & $\mathrm{~N}$ \\
\hline 3 & 401 & Lecture Theatre & $50 \times 20 \times 10$ & S & 3 & $5 \times 6$ & $\mathrm{~N}$ & 1 & $4 \times 9$ & $\mathrm{~S}$ \\
\hline 4 & - & Stationary Shop & $15 \times 12 \times 10$ & $\mathrm{~N}$ & 1 & $5 \times 6$ & $\mathrm{~N}$ & 1 & $3 \times 6$ & $\mathrm{~S}$ \\
\hline
\end{tabular}

Table 6. Dimensions of fifth floor

\begin{tabular}{|c|c|c|c|c|c|c|c|c|c|c|}
\hline \multirow[t]{2}{*}{$\mathrm{SN}$} & \multicolumn{4}{|c|}{ Room } & \multicolumn{3}{|c|}{ Window } & \multicolumn{3}{|c|}{ Door } \\
\hline & No & Type & $\begin{array}{l}\text { Dimensions lbh* } \\
\text { (In Foot) }\end{array}$ & Orientation & Quantity & $\begin{array}{l}\text { Dimensions lbh* } \\
\text { (In Foot) }\end{array}$ & Orientation & Quantity & $\begin{array}{l}\text { Dimensions } 1 b^{* *} \\
\text { (In Foot) }\end{array}$ & Orientation \\
\hline \multirow[t]{4}{*}{1} & 601 & $\begin{array}{l}\text { Miscellaneous } \\
\text { Room }\end{array}$ & $60 \times 25 \times 10$ & $\mathrm{~S}$ & 2 & $5 \times 6 \times 10$ & $\mathrm{~S}$ & \multirow[t]{4}{*}{1} & \multirow[t]{4}{*}{$4 \times 6 \times 10$} & \multirow[t]{4}{*}{ E } \\
\hline & & & & & 2 & $5 \times 6 \times 10$ & $\mathrm{~N}$ & & & \\
\hline & & & & & 5 & $5 \times 6 \times 10$ & W & & & \\
\hline & & & & & 2 & $5 \times 6 \times 10$ & $\mathrm{~N}$ & & & \\
\hline
\end{tabular}

$\mathrm{lbh}^{*}=$ length $\mathrm{x}$ breadth $\mathrm{x}$ height, $\mathrm{lb}^{* *}=$ length $\mathrm{x}$ breadth 
Under the steady state approach (which does not account the effect of heat capacity of building materials), the heat balance for room air can be written as given by [15]:

$$
\mathrm{Q}_{\text {total }}=\mathrm{Q}_{\mathrm{c}}+\mathrm{Q}_{\mathrm{s}}+\mathrm{Q}_{\mathrm{i}}+\mathrm{Q}_{\mathrm{v}}
$$

where

$\mathrm{Q}_{\text {total }}$ is total energy requirement if it is -ve then heating is required and if it is +ve then cooling is required.

$\mathrm{Q}_{\mathrm{c}}$ is conduction losses in a building, $\mathrm{Q}_{\mathrm{s}}$ is solar gain in a building, $Q_{i}$ is internal gain in a building, $Q_{v}$ is ventilation losses in a building.

\subsection{Conduction}

The rate of heat conduction $\left(\mathrm{Q}_{\mathrm{c}}\right)$ through any element such as roof, wall or floor under steady state can be written as

$$
\mathrm{Q}_{\mathrm{c}}=\mathrm{AU} \Delta \mathrm{T}
$$

where

$A=$ surface $\operatorname{area}\left(\mathrm{m}^{2}\right), \mathrm{U}=$ thermal transmittance $\left(\mathrm{W} / \mathrm{m}^{2} \mathrm{~K}\right)$, $\Delta \mathrm{T}=$ temperature difference between inside and outside air (K)

If the surface is also exposed to solar radiation then

$\Delta \mathrm{T}=\mathrm{T}_{\text {so }}-\mathrm{T}_{\mathrm{i}}$

where $T_{i}$ is the indoor temperature; $T_{s o}$ is the solar air temperature, calculated using the expression:

$\mathrm{T}_{\mathrm{so}}=\mathrm{T}_{\mathrm{o}}+\alpha \mathrm{S}_{\mathrm{T}} / \mathrm{h}_{\mathrm{o}}-\varepsilon \Delta \mathrm{R} / \mathrm{h}_{\mathrm{o}}$

where

$\mathrm{T}_{0}=$ daily average value of hourly ambient temperature $(\mathrm{K}), \alpha=$ absorptance of the surface for solar radiation, $\mathrm{S}_{\mathrm{T}}=$ daily average value of hourly solar radiation incident on the surface $\left(\mathrm{W} / \mathrm{m}^{2}\right)$

$\mathrm{h}_{\mathrm{o}}=$ outside heat transfer coefficient $\left(\mathrm{W} / \mathrm{m}_{2} \mathrm{~K}\right), \varepsilon=$ emissivity of the surface

$\Delta \mathrm{R}=$ difference between the long wavelength radiation incident on the surface from the sky and the surroundings, and the radiation emitted by a black body at ambient temperature

\subsection{Solar Heat Gain}

The solar gain through transparent elements can be written as:

$$
\mathrm{Q}_{\mathrm{s}}=\alpha_{\mathrm{s}} \Sigma \mathrm{A}_{\mathrm{i}} \mathrm{S}_{\mathrm{gi}_{\mathrm{i}}} \tau_{\mathrm{i}}
$$

where

$\alpha_{\mathrm{s}}=$ mean absorptivity of the space, $\mathrm{A}_{\mathrm{i}}=$ area of the ith transparent element $\left(\mathrm{m}^{2}\right), \mathrm{S}_{\mathrm{gi}}=$ daily average value of solar radiation (including the effect ofshading) on the ith transparent element $\left(\mathrm{W} / \mathrm{m}^{2}\right), \tau_{\mathrm{i}}=$ transmissivity of the ith transparent element.
The heat flow rate due to ventilation of air between the interior of a building and the outside depends on the rate of air exchange. It is given by:

$$
\mathrm{Q}_{\mathrm{v}}=\rho \mathrm{V}_{\mathrm{r}} \mathrm{C} \Delta \mathrm{T}
$$

where,

$\rho=$ density of air $\left(\mathrm{kg} / \mathrm{m}^{3}\right), \mathrm{V}_{\mathrm{r}}=$ ventilation rate $\left(\mathrm{m}^{3} / \mathrm{s}\right), \mathrm{C}=$ specific heat of air $(\mathrm{J} / \mathrm{kgK})$

$\Delta \mathrm{T}=$ temperature difference $(\mathrm{To}-\mathrm{Ti})(\mathrm{K})$

\subsection{Internal Gain}

The heat generated by occupants is a heat gain for the building; its magnitude depends on the level of activity of a person.

$$
\begin{array}{r}
\mathrm{Qi}=(\text { No of people } \times \text { heat output rate })+\text { Rated wattage of } \\
\text { lamps }+ \text { Appliance load (5) }
\end{array}
$$

Mean hourly values of data for various places in India are available in the handbook by [1].

Irrespective of developing a new model the neural fitting tool (nftool) of neural network of MATLAB Version 7.11.0.584 (R2010b) with 32-bit (win 32) had been used. Out of six samples four had been used for training, one sample each had been used for validation and testing.

\section{Results}

The total energy requirement during winter is calculated as (Table 7)

$\mathrm{Qm}=-1,211,228 \mathrm{~kW}$ per annum whose ANN graphs are shown in Fig 1 \& Fig 2

The total energy requirement during summer is calculated as (Table 8)

$\mathrm{Qm}=874,668 \mathrm{~kW}$ per annum whose ANN graphs are shown in Fig 3 \& Fig 4

Total Load $=$ Heating Load + Cooling Load

Total $\mathrm{Load}=2,085,896 \mathrm{~kW}$

Table 7. Total heat load in $\mathrm{kW}[19]$

\begin{tabular}{|c|c|c|c|c|c|}
\hline & $\mathrm{Q}_{\mathrm{c}}$ & $\mathrm{Q}_{\mathrm{s}}$ & $\mathrm{Q}_{\mathrm{v}}$ & $\mathrm{Q}_{\mathrm{i}}$ & $\mathrm{Q}_{\text {total }}$ \\
\hline & -53.6 & 90.6 & -232.9 & 78.8 & -117.1 \\
\hline $\begin{array}{c}\text { Annual } \\
\text { value }\end{array}$ & $-2,31,552$ & $1,14,156$ & $-10,06,128$ & $1,51,296$ & $-12,11,228$ \\
\hline
\end{tabular}

Table 8. Total cooling load in $\mathrm{kW}$ [20]

\begin{tabular}{|c|c|c|c|c|c|}
\hline & $\mathrm{Q}_{\mathrm{c}}$ & $\mathrm{Q}_{\mathrm{s}}$ & $\mathrm{Q}_{\mathrm{v}}$ & $\mathrm{Q}_{\mathrm{i}}$ & $\mathrm{Q}_{\text {total }}$ \\
\hline & 22.6 & 67.4 & 136.1 & 108.5 & 334.6 \\
\hline $\begin{array}{c}\text { Annual } \\
\text { value }\end{array}$ & 97632 & 84924 & 587952 & 104160 & 874668 \\
\hline
\end{tabular}

\subsection{Ventilation}




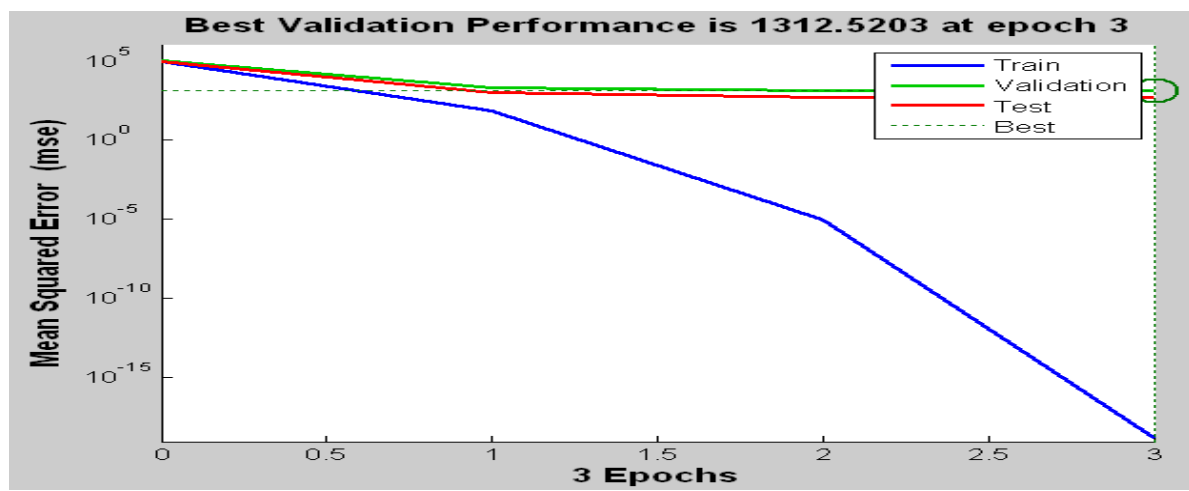

Figure 1. Validation Performance of Heat load $\left(\mathrm{Q}_{\mathrm{m}}\right)$

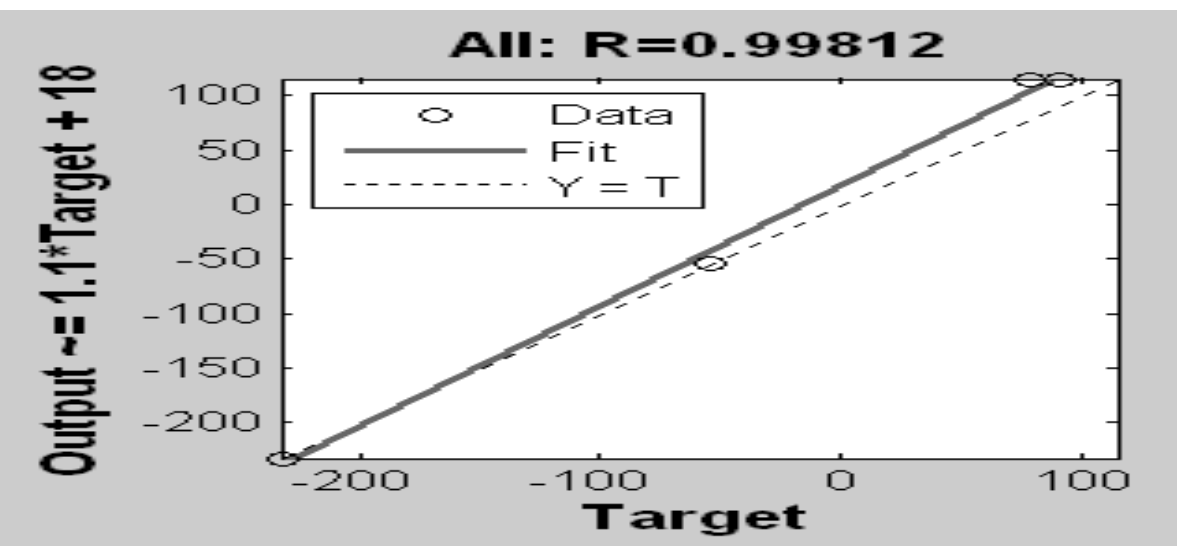

Figure 2. Regression Analysis of Heat load $\left(\mathrm{Q}_{\mathrm{m}}\right)$

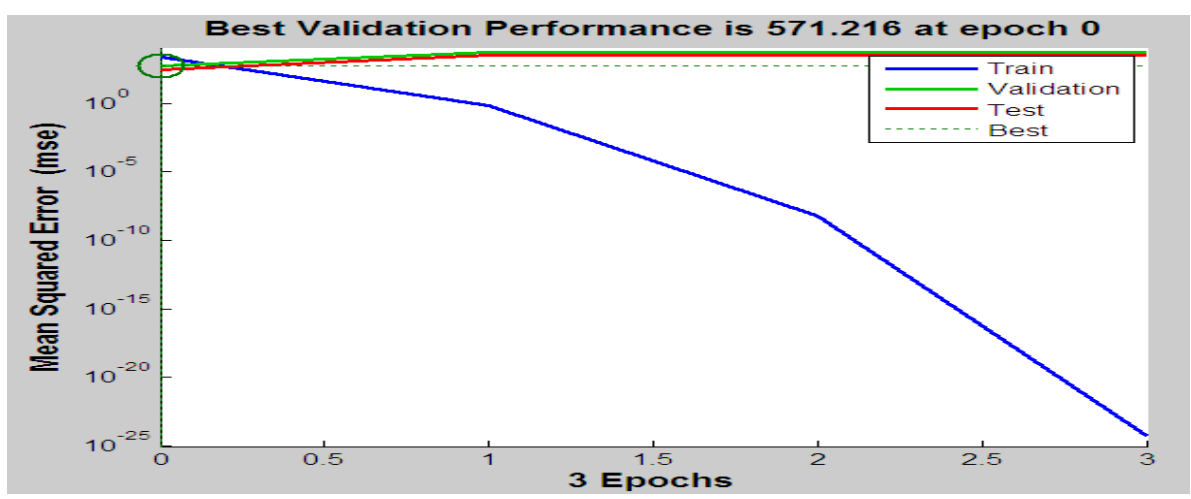

Figure 3. Validation Performance of Cooling load $\left(\mathrm{Q}_{\mathrm{m}}\right)$

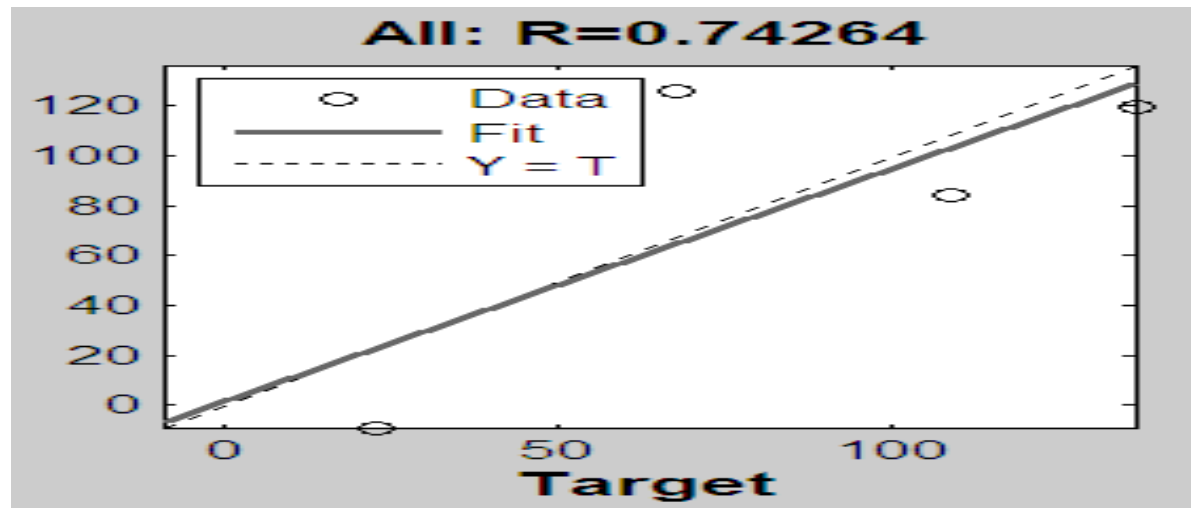

Figure 4. Regression Analysis of Cooling load $\left(\mathrm{Q}_{\mathrm{m}}\right)$ 


\section{Discussion}

In most residential buildings, optimization of thermal comfort and energy consumption is not achieved. From the above system descriptions one can see that ANNs have been applied in a wide range of fields for modeling, prediction and control of building energy systems. What is required for setting up such systems is data that represents the past history and performance of the real system and a suitable selection of ANN models. The accuracy of the selected models is tested with the data of the past history and performance of the real system. The neural network model was used with 10 hidden neurons which didn't indicate any major problem with the training. The validation and test curves were very similar. The evaluation and validation of an artificial neural network prediction model were based upon one or more selected error metrics. Generally, neural network models which perform a function approximation task will use a continuous error metric such as mean absolute error (MAE), mean squared error (MSE) or root mean squared error (RMSE). The errors will be summed over the validation set of inputs and outputs, and then normalized by the size of the validation set [16]. Here we had used mean squared error (MSE) for the best validation performance. The next step in validating the network was to create a regression plot, which showed the relationship between the outputs of the network and the targets. If the training were perfect, the network outputs and the targets would be exactly equal, but the relationship was rarely perfect in practice. The three axes represented the training, validation and testing data. The dashed line in each axis represented the perfect result outputs $=$ targets. The solid line represented the best fit linear regression line between outputs and targets. The $\mathrm{R}$ value was an indication of the relationship between the outputs and targets. If $\mathrm{R}=1$, this indicated that there was an exact linear relationship between outputs and targets. If $\mathrm{R}$ was close to zero, then there was no linear relationship between outputs and targets.

\section{Conclusions}

The study revealed that the total load of a six storey building is 2.09 million $\mathrm{kW}$ thus, heating in winter and cooling in summer is required to meet out this energy demand. If we use electricity it will produce 8.34 ton carbon per annum and the cost of electricity used will be $\$ 53,776.16$ (Table 9). If we use diesel to meet out this energy requirement then 563.19 ton of carbon will be emitted and it will cost \$ 139,184.62 [17]. The above results necessitate the use of solar passive technologies to meet out this energy requirement during winters and summers. Increasing awareness of environmental issues has led to development of a large number of energy conservation technologies for buildings, especially in more developed countries [18]. Energy savings potential (ESP) is a very important indicator for developing these technologies.
Table 9. Carbon emission and cost [17]

\begin{tabular}{|c|c|c|c|c|}
\hline Fuel & $\begin{array}{c}\text { Carbon } \\
\text { Emission } \\
\text { per kWh (in } \\
\mathrm{g} \text { ) }\end{array}$ & $\begin{array}{c}\text { Total } \\
\text { Carbon } \\
\text { Emission } \\
\text { (in ton) }\end{array}$ & $\begin{array}{c}\text { Fuel } \\
\text { Required }\end{array}$ & $\begin{array}{c}\text { Total cost } \\
\text { (in USD) }\end{array}$ \\
\hline Electricity & 4 & 8.34 & - & 53776.16 \\
\hline Diesel & 270 & 563.19 & 167302.8 & 139184.62 \\
\hline $\begin{array}{c}\text { Solar } \\
\text { Energy }\end{array}$ & 0 & 0 & 0 & - \\
\hline
\end{tabular}

\section{Limitations}

ANN models like all other approximation techniques have relative advantages and disadvantages. There are no rules as to when this particular technique is more or less suitable for an application. Result of ANN depends upon number of hidden layer neurons. In order to get optimize result we should select optimize number of hidden layer neurons. One way of selecting hidden layer neuron using optimize algorithm technique and other way is hit and trial method. In existing proposed model hit and trial method has been used but it is never easy to comment that the used number of hidden neurons is perfect.

\section{REFERENCES}

[1] A. Mani and S. Rangarajan. Solar radiation over India, Allied Publishers, New Delhi, 1982.

[2] S. A. Kalogirou. Artificial neural networks in energy applications in buildings, International Journal of Low-Carbon Technologies, Vol. 1. No. 3, 201-16, 2006.

[3] S. A. Kalogirou, C. C. Neocleous and C. N. Schizas. Building heating load estimation using artificial neural networks, Proceedings of the 17 th international conference on parallel architectures and compilation techniques, Toronto, Canada, October, 2008.

[4] B. B. Ekici and U. T. Aksoy. Prediction of building energy consumption by using artificial neural networks, Advances in Engineering Software, Vol. 40, No. 5, 356-62, 2009.

[5] T. Olofsson and S. Andersson. Long-term energy demand predictions based on short-term measured data, Energy and Buildings, Vol. 33, No. 2, 85-91, 2001.

[6] R. Yokoyama, T. Wakui and R. Satake. Prediction of energy demands using neural network with model identification by global optimization, Energy Conversion and Management, Vol. 50, No. 2, 319-27, 2009.

[7] J. F. Kreider, D. E. Claridge, P. Curtiss, R. Dodier, J. S. Haberl and M. Krarti. Building energy use prediction and system identification using recurrent neural networks, Journal of Solar Energy Engineering, Vol. 117, No. 3, 161-6, 1995.

[8] A. E. Ben-Nakhi and M. A. Mahmoud. Cooling load prediction for buildings using general regression neural network, Energy Conversion and Management, Vol. 45, No. 
13, 2127-41, 2004.

[9] S. A. Kalogirou and M. Bojic. Artificial neural networks for the prediction of the energy consumption of a passive solar building, Energy, Vol. 25, No. 5, 479-91, 2000.

[10] C. W. Yan and J. Yao. Application of ANN for the prediction of building energy consumption at different climate zones with HDD and CDD, Proceedings of the 2nd international conference on future computer and communication, Wuhan, China, May, 2010.

[11] S. L. Wong, K. K. W. Wan and T. N. T. Lam. Artificial neural networks for energy analysis of office buildings with day lighting, Applied Energy, Vol. 87, No. 2, 551-7, 2010.

[12] M. Aydinalp, V. I. Ugursal and A. S. Fung. Modeling of the appliance, lighting, and space cooling energy consumptions in the residential sector using neural networks, Applied Energy, Vol. 71 No. 2, 87-110, 2002.

[13] S. K. Sheikh and M. G. Unde. Short Term Load Forecasting using ANN Technique, International Journal of Engineering Sciences \& Emerging Technologies, Vol. 1, No. 2, 27-107, 2012.

[14] S. Karatasou, M. Santamouris and V. Geros. Modeling and predicting building's energy use with artificial neural networks: methods and results, Energy and Buildings, Vol. 38, No. 8, 949-58, 2006.
[15] J. K. Nayak and J. A. Prajapati. Handbook on Energy Conscious Buildings, Prepared under the interactive R \& D project no. 3/4(03)/99-SEC between Indian Institute of Technology, Bombay and Solar Energy Centre, Ministry of Non-conventional Energy Sources, Government of India, May, 2006.

[16] N. Kartam, I. Flood and J. Garrett. Fundamentals and Applications, ASCE Press, 1996.

[17] Volker Quaschning, Regenerative Energiesysteme, Printed in Germany, 2011. http://www.volker-quaschning.de/datserv/CO2-spez/index_e .php

[18] M. Chikada, T. Inou et al. Evaluation of Energy Saving Methods in a Research Institute Building, CCRH. PLEA2001-The 18th Conference on Passive and Low Energy Architecture, Florianopolis-BRAZIL, 883-888, 2001.

[19] Rajesh Kumar, R. K. Aggarwal and J. D. Sharma. Predicting energy requirement for heating the building using artificial neural network, International Journal of Developmental Research, Vol. 3, No. 5, 14-19, 2013.

[20] Rajesh Kumar, R. K. Aggarwal, J. D. Sharma and S. Pathania. Predicting energy requirement for cooling the building using artificial neural network, Journal of Technology innovations in Renewable Energy, Vol. 1, No.1, 113-121, 2012. 